\title{
Reno-protective effects of renin-angiotensin system blockade in type 2 diabetic patients: a systematic review and network meta-analysis
}

\author{
P. Vejakama • A. Thakkinstian • D. Lertrattananon • \\ A. Ingsathit • C. Ngarmukos • J. Attia
}

Received: 9 September 2011 / Accepted: 7 November 2011 /Published online: 22 December 2011

(C) The Author(s) 2011. This article is published with open access at Springerlink.com

\begin{abstract}
Aims/hypothesis This meta-analysis aimed to compare the renal outcomes between ACE inhibitor (ACEI)/angiotensin II receptor blocker (ARB) and other antihypertensive drugs or placebo in type 2 diabetes.

Methods Publications were identified from Medline and Embase up to July 2011. Only randomised controlled trials comparing ACEI/ARB monotherapy with other active drugs
\end{abstract}

Electronic supplementary material The online version of this article (doi:10.1007/s00125-011-2398-8) contains peer-reviewed but unedited supplementary material, which is available to authorised users.

P. Vejakama $\cdot$ A. Thakkinstian $(\bowtie) \cdot A$. Ingsathit

Section for Clinical Epidemiology and Biostatistics,

Faculty of Medicine, Ramathibodi Hospital, Mahidol University,

Rama 6 Road, Rachatevi,

Bangkok 10400, Thailand

e-mail: raatk@mahidol.ac.th

P. Vejakama

Bundarik Hospital,

Ubon Ratchathani Province, Thailand

D. Lertrattananon

Department of Family Medicine, Faculty of Medicine,

Ramathibodi Hospital, Mahidol University,

Bangkok, Thailand

C. Ngarmukos

Division of Endocrinology and Metabolism,

Department of Medicine, Faculty of Medicine,

Ramathibodi Hospital, Mahidol University,

Bangkok, Thailand

\section{J. Attia}

Centre for Clinical Epidemiology and Biostatistics,

School of Medicine and Public Health,

University of Newcastle and Hunter Medical Research Institute,

Newcastle, NSW, Australia or placebo were eligible. The outcome of end-stage renal disease, doubling of serum creatinine, microvascular complications, microalbuminuria, macroalbuminuria and albuminuria regression were extracted. Risk ratios were pooled using a random-effects model if heterogeneity was present; a fixedeffects model was used in the absence of heterogeneity.

Results Of 673 studies identified, 28 were eligible $(n=13-$ 4,912). In direct meta-analysis, ACEI/ARB had significantly lower risk of serum creatinine doubling (pooled $\mathrm{RR}=0.66$ [95\% CI $0.52,0.83]$ ), macroalbuminuria (pooled $\mathrm{RR}=0.70$ $[95 \% \mathrm{CI} 0.50,1.00]$ ) and albuminuria regression (pooled RR $1.16[95 \%$ CI $1.00,1.39])$ than other antihypertensive drugs, mainly calcium channel blockers (CCBs). Although the risks of end-stage renal disease and microalbuminuria were lower in the ACEI/ARB group (pooled RR 0.82 [95\% CI 0.64, 1.05] and 0.84 [95\% CI $0.61,1.15]$, respectively), the differences were not statistically significant. The ACEI/ARB benefit over placebo was significant for all outcomes except microalbuminuria. A network meta-analysis detected significant treatment effects across all outcomes for both active drugs and placebo comparisons.

Conclusions/interpretation Our review suggests a consistent reno-protective effect of ACEI/ARB over other antihypertensive drugs, mainly CCBs, and placebo in type 2 diabetes. The lack of any differences in BP decrease between ACEI/ARB and active comparators suggest this benefit is not due simply to the antihypertensive effect.

Keywords Albuminuria regression - Angiotensin receptor blockers - Angiotensin-converting enzyme inhibitors .

Doubling of serum creatinine - End-stage renal disease .

Macroalbuminuria $\cdot$ Meta-analysis $\cdot$ Microalbuminuria .

Renin-angiotensin system blockade $\cdot$ Reno-protective effect . Systematic review . Type 2 diabetes 


$\begin{array}{ll}\text { Abbreviations } \\ \text { ACEI } & \text { ACE inhibitor } \\ \text { ARB } & \text { Angiotensin II receptor blocker } \\ \text { BB } & \text { Beta-blocker } \\ \text { CCB } & \text { Calcium-channel blocker } \\ \text { DBP } & \text { Diastolic BP } \\ \text { d-CCB } & \text { Dihydropyridine CCB } \\ \text { ESRD } & \text { End-stage renal disease } \\ \text { nd-CCB } & \text { Non-dihydropyridine CCB } \\ \text { RAS } & \text { Renin-angiotensin system } \\ \text { RCT } & \text { Randomised controlled trial } \\ \text { SBP } & \text { Systolic BP } \\ \text { UKPDS } & \text { UK Prospective Diabetes Study }\end{array}$

\section{Introduction}

Diabetic nephropathy is a significant health and economic burden across the world. The prevalence of micro- and macroalbuminuria in type 2 diabetes is as high as $37-40 \%$ in western countries and 57.4-59.8\% in Asian countries [1-3]. Microalbuminuria is a well-established risk factor for cardiovascular disease and is also associated with end-stage renal disease (ESRD) [4-7]. Preventive treatments have been prescribed for type 2 diabetes with or without hypertension with the aim of lowering BP, and delaying or even preventing the progression of diabetic nephropathy.

The reno-protective effects of renin-angiotensin system (RAS) blockers in type 2 diabetes have been controversial [8]. A few systematic reviews have been conducted [9-11], but these reviews pooled studies with mixed populations of participants with type 1 and type 2 diabetes, with and without diabetic nephropathy, and the focus was mainly on surrogate rather than clinical outcomes. We therefore conducted a systematic review and meta-analysis comparing the effects of $\mathrm{ACE}$ inhibitor (ACEI)/angiotensin II receptor blocker (ARB) with other antihypertensive drugs and placebo on ESRD, doubling of serum creatinine, microvascular complications, micro- and macroalbuminuria and regression of albuminuria.

\section{Methods}

Study selection

English-language publications were identified from Medline (1949-July 2011) and Embase (1974-April 2011) using PubMed and Ovid search engines. Search terms and strategies for Medline were as follows: (type 2 diabetes or type 2 diabetes mellitus or type $2 \mathrm{DM}$ or non insulin dependent diabetes) and (ACEI or angiotensin-converting enzyme inhibitors or ACE inhibitors or ARB or angiotensin receptor blockers*) and (nephropathy or overt nephropathy or microalbuminuria or macroalbuminuria or microvascular complications or doubling serum creatinine or ESRD or end-stage renal disease or urinary albumin excretion). We also searched for any additional studies in the reference lists of identified publications. Data from the most recent or the most complete report by the same authors were used. We restricted our searches to clinical trials. Studies with the following criteria were included: type 2 diabetic adult individuals, randomised trial comparing any single ACEI/ARB with other single agents (e.g. beta-blocker [BB], calciumchannel blocker [CCB], diuretics) or placebo, had at least one of the following outcomes (microalbuminuria, macroalbuminuria, albuminuria regression, microvascular complications, serum creatinine doubling and/or ESRD), and reported number of patients and events in each treatment arm. Studies were excluded if they were crossover trials, used dual therapies or compared different dosages of ACEI/ARB.

Outcome measures The outcomes of interest were ESRD, doubling of serum creatinine, microvascular complications, macroalbuminuria, microalbuminuria and regression of albuminuria. ESRD was defined as a requirement for renal replacement therapy or dialysis. Doubling of serum creatinine was defined as an increase in serum creatinine level of at least two times compared with baseline level. Microvascular complications were defined as a composite of having nephropathy and/or retinopathy. Microalbuminuria was defined as urine albumin excretion rate of $30-300 \mathrm{mg} / 24 \mathrm{~h}$ for $24 \mathrm{~h}$ urine collection, $3.5-35 \mathrm{mg} / \mathrm{mmol}$ for urinary albumin/ creatinine ratio from a spot urine collection, or $20-199 \mu \mathrm{g} / \mathrm{min}$ for timed urine collection. Macroalbuminuria was defined as urine albumin excretion rate $\geq 300 \mathrm{mg} / 24 \mathrm{~h}, \geq 25 \mathrm{mg} / \mathrm{mmol}$ creatinine or $\geq 200 \mu \mathrm{g} / \mathrm{min}$ for the same specimens, respectively. Regression of albuminuria was defined as a change from a higher to a lower stage of albuminuria.

\section{Data extraction}

Two investigators (P. Vejakama and D. Lertrattananon) independently extracted data, including study and participant characteristics (e.g. age, BP, albuminuria stage, serum glucose, and $\mathrm{HbA}_{1 \mathrm{c}}$ ) and numbers of events across intervention groups. Discrepancies were discussed with a third party (A. Thakkinstian) and resolved by consensus.

Risk of bias assessment

Risk of bias was assessed using the Cochrane Collaboration's tool addressing six domains: sequence generation, allocation concealment, blinding of participants/outcome assessors, incomplete outcome, selective outcome reporting and other source of bias. Two investigators (P. Vejakama and 
D. Lertrattananon) independently completed the assessments; discrepancies were discussed with a third party (A. Thakkinstian) and resolved by consensus.

\section{Statistical analysis}

For direct meta-analysis, the intervention of interest was ACEI/ARB monotherapy, while the comparator group was any antihypertensive drug or placebo. Pooled RRs were used to compare treatment effects. The $Q$ test and $I^{2}$ statistic were used to assess the presence and degree of heterogeneity. If heterogeneity was present or $I^{2}>25 \%$, the random-effects model was applied, otherwise the fixed-effects model was used. Sources of heterogeneity were explored using metaregression. The Harbord test and a funnel plot were applied to assess publication bias [12]. If either suggested asymmetry of the funnel, contour-enhanced funnel plots were used to distinguish the cause of asymmetry (i.e. heterogeneity and reporting bias) $[13,14]$.

A network meta-analysis [15-17] was performed to compare indirectly all treatment effects. Summary data for treatments and outcomes were expanded to the individual patient level. A binary regression analysis was applied and the pooled RRs and 95\% CIs were estimated. All analyses were performed using STATA version 11 (Stata Corp., College Station, TX, USA). $p$ values less than 0.05 were considered statistically significant, except for the test of heterogeneity where $p<0.10$ was used.

\section{Results}

Of the 673 articles located, full papers of 153 plus four additional studies from reference lists were reviewed, leading to data pooling for 28 studies (see Fig. 1). The characteristics of these studies are described in Table 1. The major ACEI/ ARBs used were enalapril (32\%), followed by lisinopril $(10.7 \%)$ and ramipril $(10.7 \%)$. The majority of comparators were dihydropyridine CCBs (d-CCBs; 43\%), placebo $(39 \%)$, or $\mathrm{BB} /$ diuretic/non-dihydropyridine $\mathrm{CCBs}$ (ndCCBs; 18\%). Numbers of individuals are summarised according to the treatments and outcomes of interest (electronic supplementary material [ESM] Tables 1-6).

The assessments of risk of bias are described in ESM Table 7. Problems with incomplete outcome reports were least frequent in $2 / 28(7.1 \%)$ studies [18, 19], followed by other sources of bias in 5/28 (17.9\%) [18, 20-23] and selective reporting of outcomes in $5 / 28(17.9 \%)[18,21$, 22, 24, 25]. Although all studies were randomised controlled trials (RCTs), only 14 (50.0\%) studies clearly described randomisation [21, 25-37] and allocation concealment [21, 22, 26-29, 31, 33-39].
Direct meta-analysis

ESRD Nine studies [20, 21, 24, 26, 29, 30, 33, 34, 40] reported the effect of ACEI/ARB on ESRD $(n=13,295)$. Of these, five studies [20, 21, 26, 30, 40] compared ACEI with other antihypertensive drugs (three BB, two d-CCB), three [24, 29, 34] compared ACEI with placebo, and one [33] compared ARB with d-CCB and placebo (ESM Table 1).

Six studies [20, 21, 26, 30, 33, 40] directly compared ACEI/ARB with other active drugs $(n=1,090$ vs 1,055$)$; four were trials that studied patients with macroalbuminuria whereas the other two studied mixed patients with microand macroalbuminuria.

The treatment effects were homogeneous $\left(\chi^{2}=1.54, d f=5\right.$, $p=0.908, I^{2}=0.0 \%$ ), suggesting that ACEI/ARB reduced the risk of $\mathrm{ESRD}$ by $18 \%$ (pooled $\mathrm{RR}=0.82$ [95\% CI 0.64 , 1.05]; see Fig. 2a). However, this result was not statistically significant (Table 2). Neither the Harbord test nor a funnel plot (ESM Fig. 1a) suggested publication bias (coefficient $-0.001, \mathrm{SE}=0.436, p=0.998)$.

For placebo controls, pooled estimates based on four studies [24, 29, 33, 34] ( $n=5,581$ vs 5,569) demonstrated homogeneous treatment effects $\left(\chi^{2}=1.11, d f=3, p=0.774\right.$, $I^{2}=0.0 \%$; Fig. 2b) despite a mix of patients with normo-, micro- and macroalbuminuria. ACEI/ARB significantly decreased the risk of ESRD by $20 \%$ (pooled $\mathrm{RR}=0.80[95 \%$ CI 0.69, 0.93]; Table 2). Although the Harbord test was not significant (coefficient 1.220, $\mathrm{SE}=0.311, p=0.059$ ), the contour-enhanced funnel plot showed asymmetry (ESM Fig. 1b). One high-precision study fell in the significant area (grey shading) whereas the other three (one high and two low precision) were in the non-significant area. Applying 'trim and fill' suggested two low-precision studies (square symbols) were missing; adding these studies yielded a pooled RR of 0.78 (95\% CI $0.68,0.91)$ with $I^{2}=0 \%$.

Doubling of serum creatinine Six studies [20, 24, 29, 33, $34,39]$ reported the effect of $\mathrm{ACEI} / \mathrm{ARB}$ vs other antihypertensive drugs on doubling of serum creatinine $(n=16,216)$, and five studies [24, 29, 33, 34, 39] reported ACEI/ARB vs placebo (ESM Table 2).

The treatment effects of ACEI/ARB vs antihypertensive drugs ( $n=597$ vs 601$)$ were homogeneous $\left(\chi^{2}=0.76, d f=1\right.$, $\left.p=0.382, I^{2}=0.0 \%\right)$ with a pooled RR of 0.66 (95\% CI 0.53 , 0.83 ; Fig. 2b), suggesting a significant reduction in risk of $34 \%$.

The ACEI/ARB effects were also present when compared with placebo $(n=7,831$ vs 7,784$)$. The pooled RR was $0.76(95 \%$ CI $0.69,0.84)$, indicating a $24 \%$ lower risk of serum creatinine doubling. The pooled effect was homogeneous $\left(\chi^{2}=1.67, d f=4, p=0.796, I^{2}=0.0 \%\right)$ without 


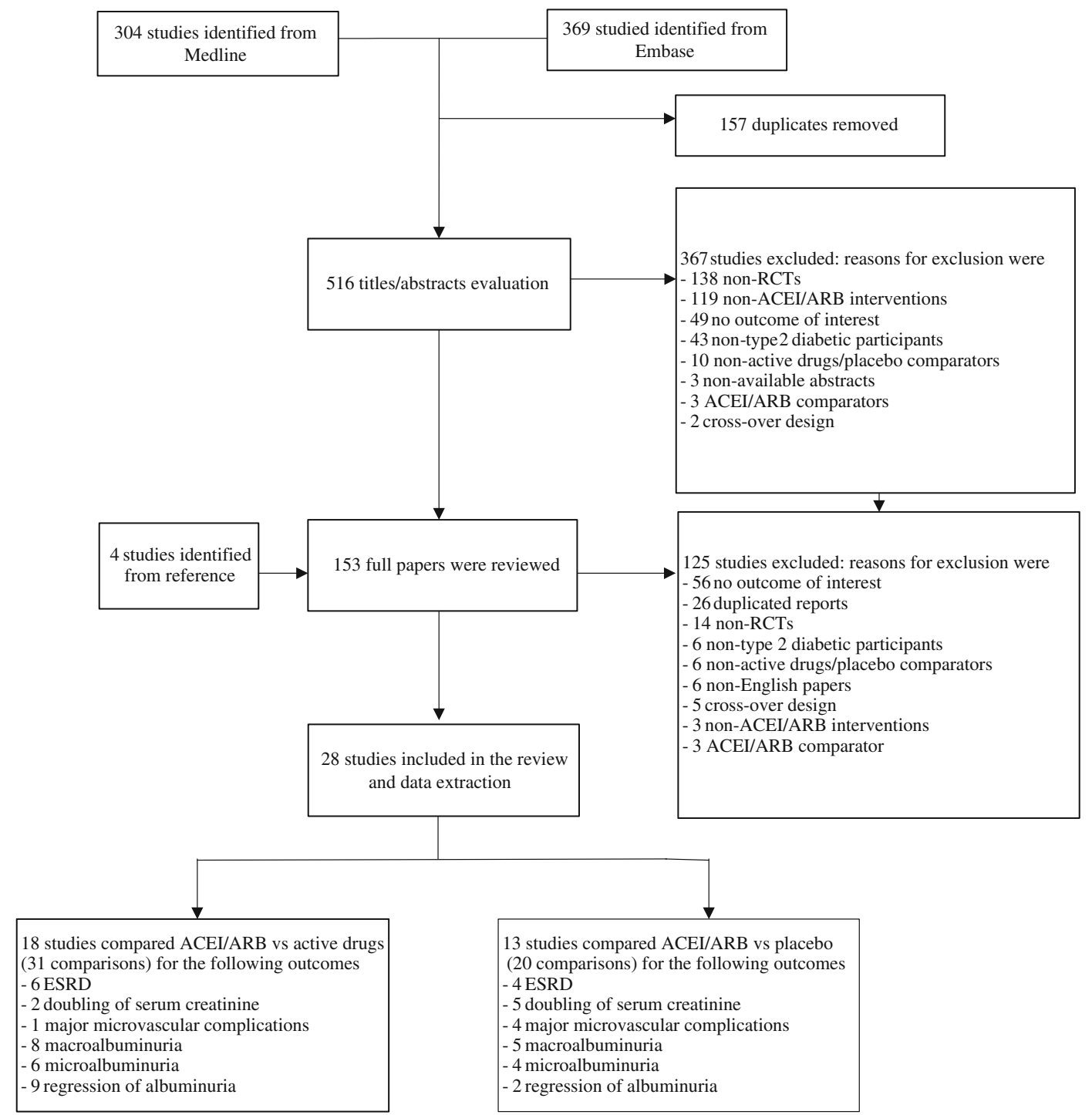

Fig. 1 Flow of study selection

publication bias (Harbord test coefficient $=0.629, \mathrm{SE}=0.908$, $p=0.538$; ESM Fig. 2).

Microvascular complications Of five studies [24, 26, 31, $32,37](n=6,489)$, only one [26] compared ACEI/ARB with active drug; the other four [24, 31, 32, 37] compared ACEI/ARB with placebo (ESM Table 3).

Compared with placebo controls $(n=2,847$ vs 2,884$)$, ACEI/ARB significantly reduced the risk of microvascular complications by $15 \%$ (pooled $\mathrm{RR}=0.85$ [95\% CI 0.76 , $0.97])$, with low heterogeneity $\left(\chi^{2}=3.34, d f=3, p=0.342\right.$, $I^{2}=10.3 \%$; Fig. 2c) and without publication bias (Harbord test coefficient $=-1.51, \mathrm{SE}=0.53, p=0.105$; ESM Fig. 3 ). In addition, the ACEI/ARB group $(n=2,884)$ had a significantly lower risk of retinopathy ( $13 \%$ lower, pooled $\mathrm{RR}=0.87$
[95\% CI 0.76, 0.99]) with low heterogeneity $\left(\chi^{2}=3.51\right.$, $\left.d f=3, p=0.319, I^{2}=14.6 \%\right)$.

Macroalbuminuria Twelve studies [19, 21-26, 32, 36, 38, 41, 42] reported ACEI/ARB effects on macroalbuminuria $(n=5,151)$ with ACEI/ARB vs other therapies (five with $\mathrm{d}-\mathrm{CCB}$, one with diuretic, one with $\mathrm{BB}$ and one with $\mathrm{BB} /$ diuretic) in eight studies $[19,21-23,25,26,36,38]$ and placebo in five studies $[24,32,36,41,42]$ (ESM Table 4). Eight trials [19, 22, 25, 26, 32, 36, 38, 41] $(n=1,401)$ studied patients with microalbuminuria, and four [21, 23, $24,42](n=3,761)$ studied mixed patients with normo- and microalbuminuria.

Direct pooled estimates of treatment effects between ACEI/ARB and other antihypertensive drugs ( $n=641$ vs 


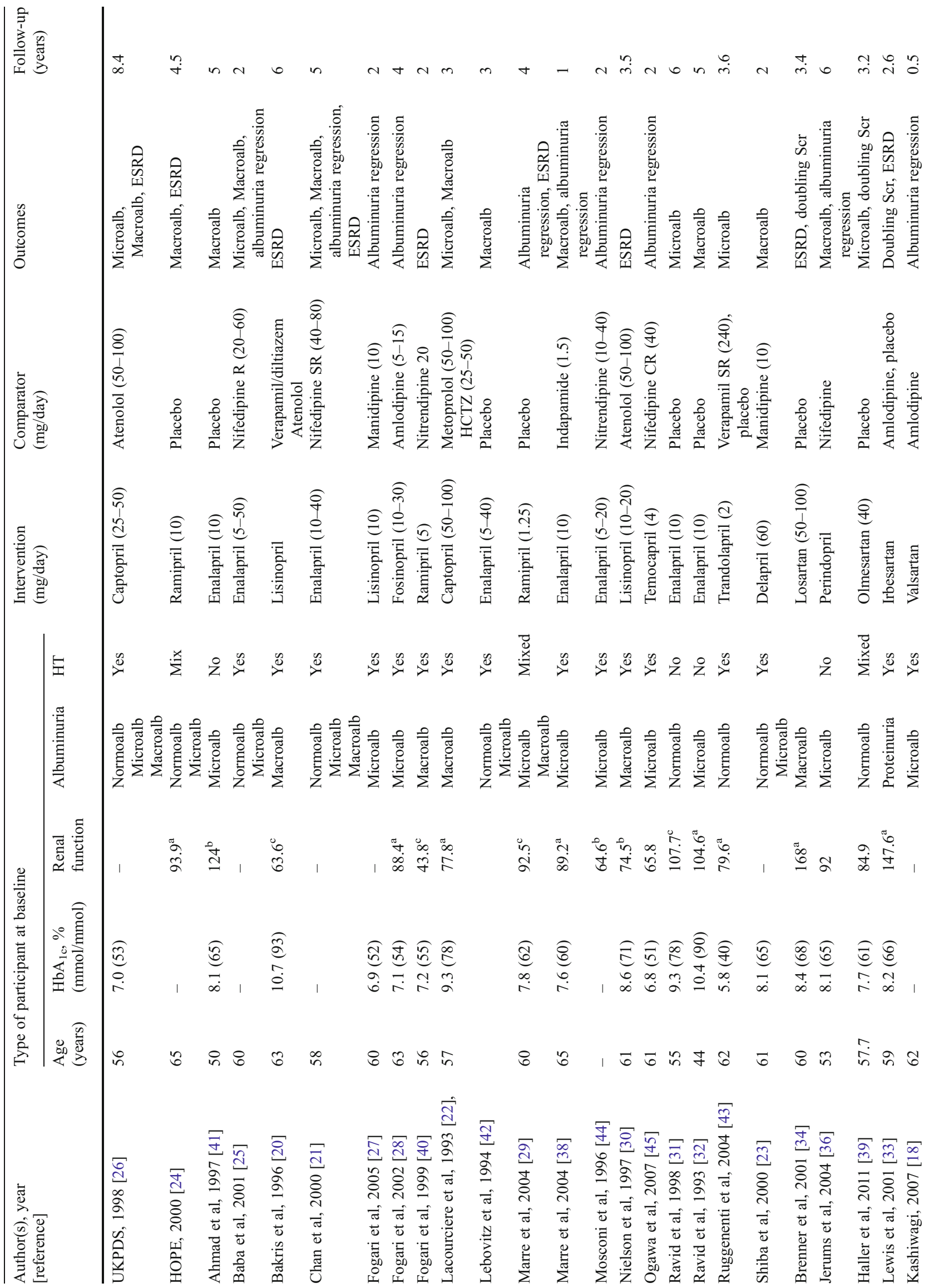




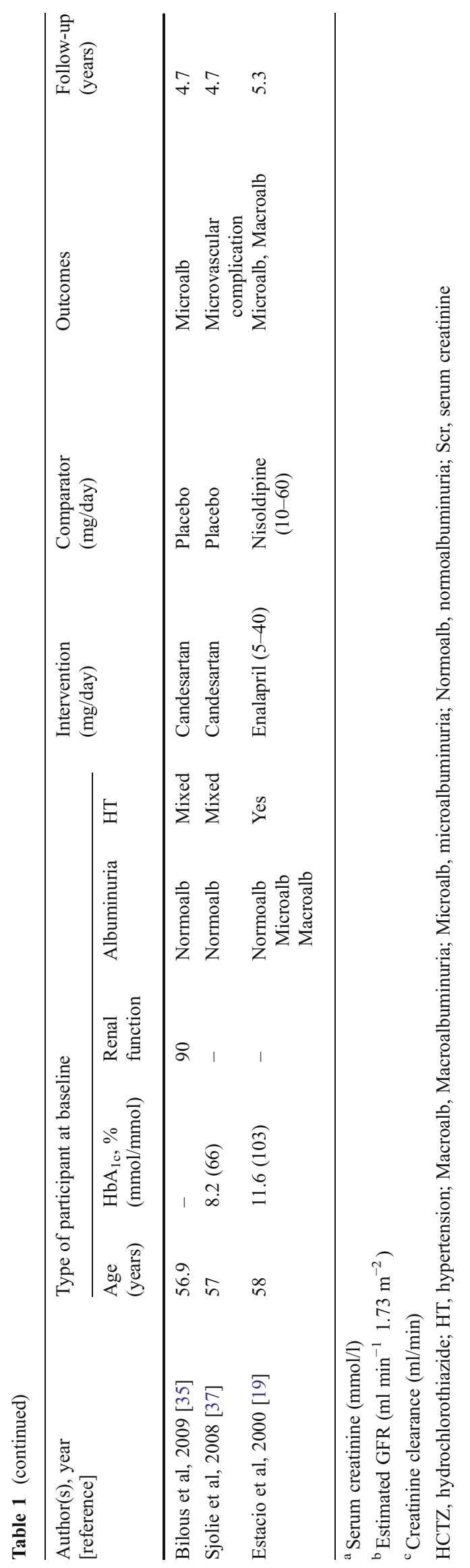


a

Authors [ref.]
Events Events

RR (95\% CI) treatment control Weight (\%)

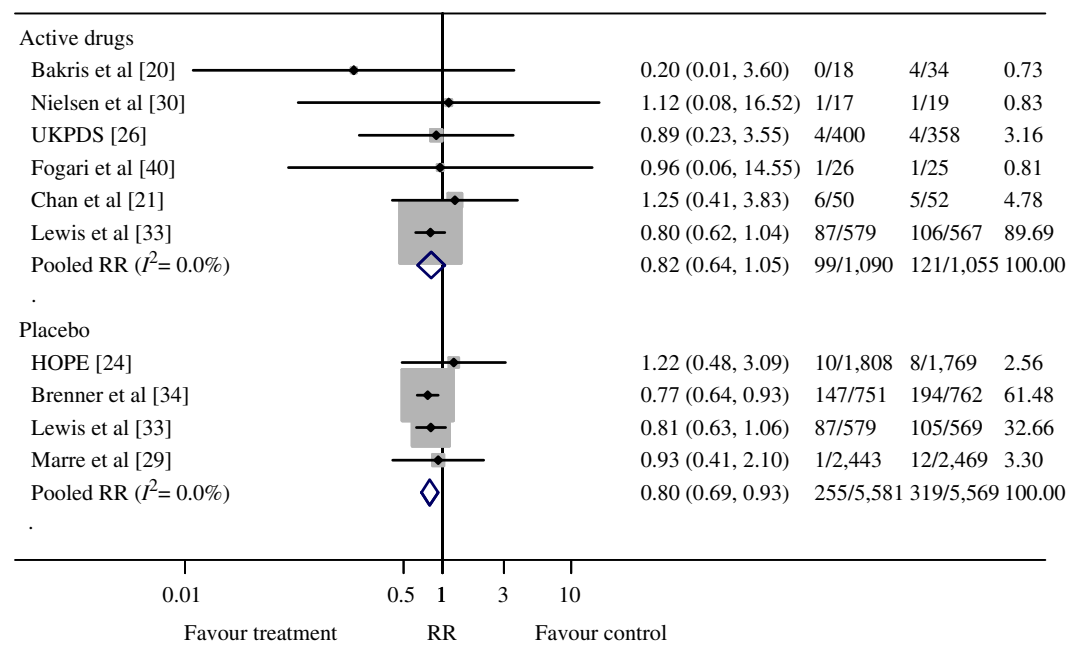

b

Authors [ref.]
Events Events

RR (95\% CI) treatment control Weight (\%)

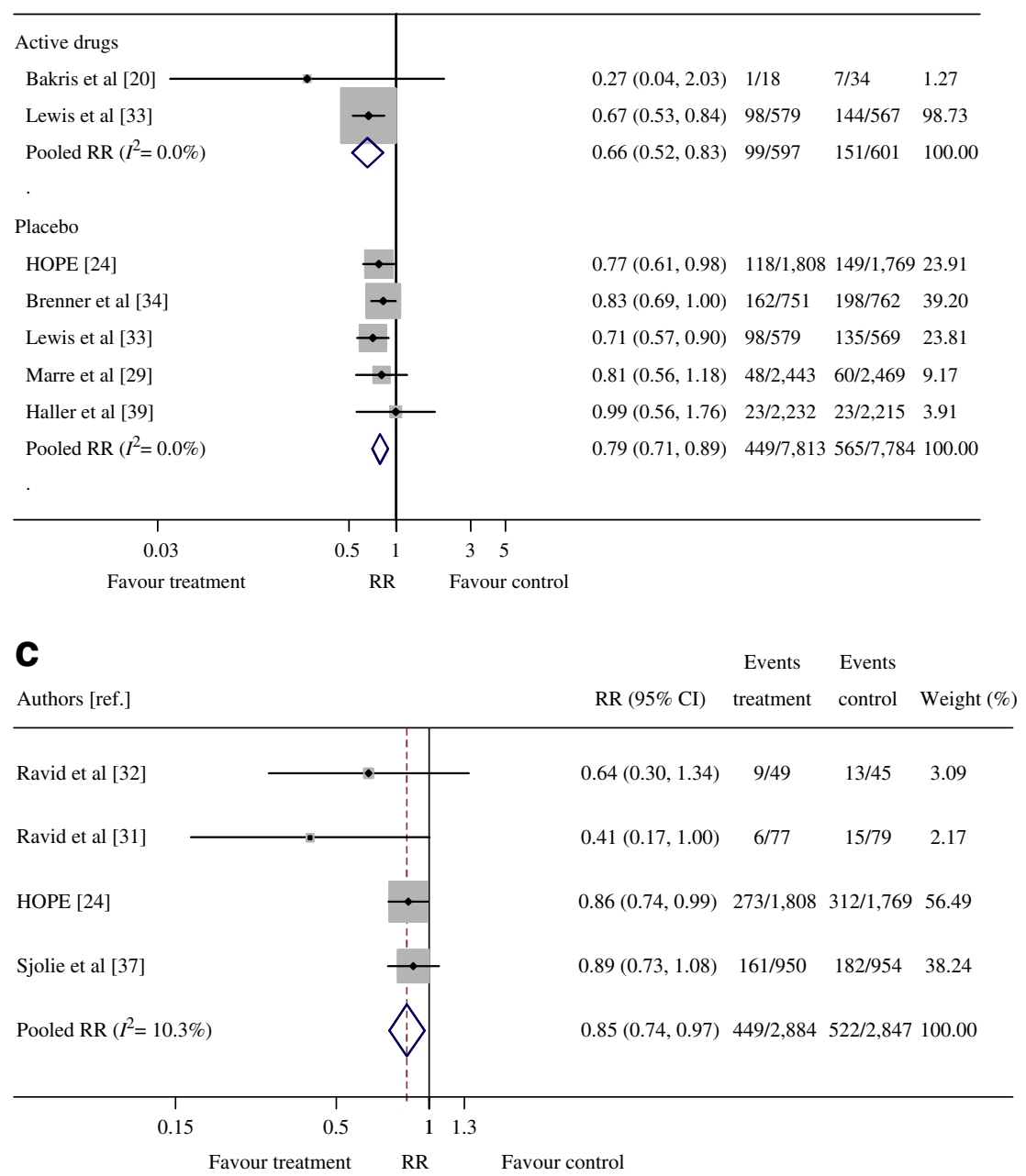

Fig. 2 Forest plots of treatment effects between ACEIs and other active drugs: (a) ESRD; (b) doubling of serum creatinine; (c) major microvascular complications; (d) macroalbuminuria; (e) microalbuminuria; and (f) albuminuria regression 
d

Authors [ref.]

Events Events

RR (95\% CI) treatment control Weight (\%)

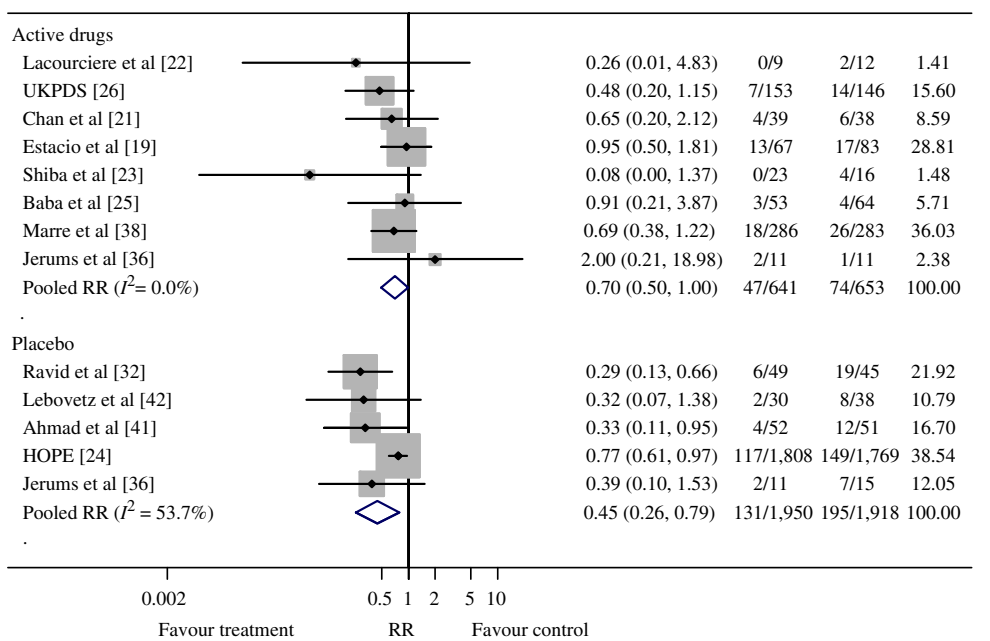

e

Authors [ref.]

Events Events

Active drugs

Lacourciere et al [22]

UKPDS [26]

Chan et al [21]

Estacio et al [19]

Baba et al [25]

Ruggenenti et al [43]

Pooled RR $\left(I^{2}=35.1 \%\right)$

Placebo

Ravid et al [31]

Ruggenenti et al [43]

Bilous et al [35]

Haller et al [39]

Pooled RR $\left(I^{2}=49.4 \%\right)$

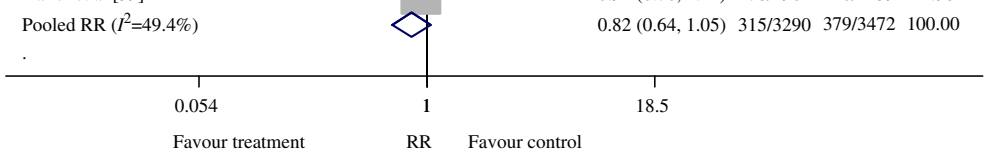

f

Authors [ref.]

Events Events

$\begin{array}{llll}0.56(0.05,5.81) & 1 / 25 & 2 / 28 & 1.74\end{array}$

$\begin{array}{llll}1.21(0.84,1.73) & 48 / 153 & 38 / 146 & 30.58\end{array}$

$\begin{array}{lllll}1.11(0.35,3.57) & 4 / 18 & 5 / 25 & 6.35\end{array}$

$\begin{array}{llll}0.87(0.53,1.42) & 25 / 123 & 25 / 107 & 22.79\end{array}$

$\begin{array}{llll}0.73(0.40,1.31) & 15 / 95 & 23 / 106 & 18.30\end{array}$

$\begin{array}{llll}0.50(0.29,0.87) & 18 / 301 & 36 / 303 & 20.24\end{array}$

$\begin{array}{llll}0.84(0.61,1.15) & 111 / 715 & 129 / 715 & 100.00\end{array}$

$\begin{array}{llll}0.34(0.13,0.90) & 5 / 77 & 15 / 79 & 5.87\end{array}$

$\begin{array}{llll}0.60(0.34,1.05) & 18 / 301 & 30 / 300 & 14.35\end{array}$

$\begin{array}{llll}0.92(0.73,1.17) & 114 / 951 & 124 / 954 & 37.21\end{array}$

$\begin{array}{lllll}0.92(0.76,1.12) & 178 / 1961 & 210 / 2139 & 42.58\end{array}$

$\begin{array}{llll}0.82(0.64,1.05) & 315 / 3290 & 379 / 3472 & 100.00\end{array}$

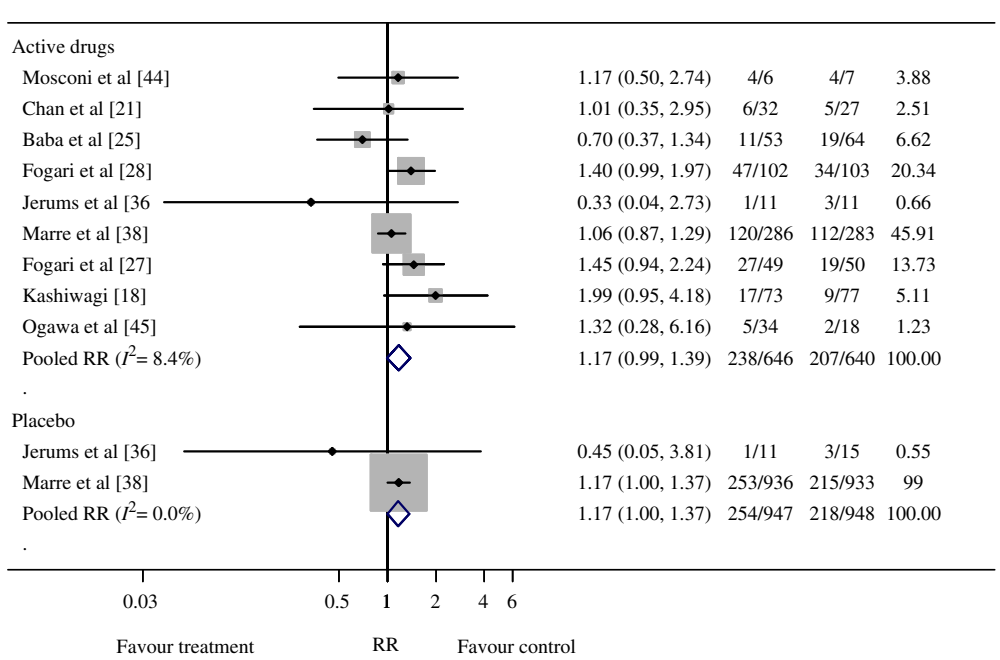

Fig. 2 (continued) 
Table 2 Summarised characteristics of studies and direct pooling treatment effects according to clinical outcomes

\begin{tabular}{llll}
\hline Dichotomous outcomes & Number of studies & Sample size & RR $(95 \%$ CI $)$ \\
\hline ESRD & & & \\
ACEI/ARB vs other active drugs & 6 & 2,147 & $0.82(0.64,1.05)$ \\
ACEI/ARB vs placebo & 4 & 10,581 & $0.80(0.69,0.93)$ \\
Doubling of serum creatinine & & & \\
ACEI/ARB vs other active drugs & 2 & 1,198 & $0.66(0.53,0.83)$ \\
ACEI/ARB vs placebo & 4 & 10,594 & $0.76(0.69,0.84)$ \\
Major microvascular complications & & & $1.28(0.81,2.03)$ \\
ACEI/ARB vs other active drugs & 1 & 758 & $0.85(0.74,0.97)$ \\
ACEI/ARB vs placebo & 4 & 6,489 & $0.71(0.50,1.00)$ \\
Macroalbuminuria & & & $0.67(0.54,0.83)$ \\
ACEI/ARB vs other active drugs & 8 & 1,211 & \\
ACEI/ARB vs placebo & 5 & 3,868 & $0.84(0.61,1.15)$ \\
Microalbuminuria & & & $0.82(0.64,1.05)$ \\
ACEI/ARB vs other active drugs & 6 & 1,430 & $1.16(0.99,1.39)$ \\
ACEI/ARB vs placebo & 4 & 6,762 & $1.17(1.00,1.37)$ \\
Albuminuria regression & & 1,286 & \\
ACEI/ARB vs other active drugs & 9 & 1,238 & \\
ACEI/ARB vs placebo & 2 & &
\end{tabular}

$653)$ of eight trials $[19,21-23,25,26,36,38]$ were homogeneous $\left(\chi^{2}=5.24, d f=7, p=0.631, I^{2}=0.0 \%\right.$; Fig. $\left.2 \mathrm{~d}\right)$ with borderline significant risk reduction of $29 \%$ (pooled $\mathrm{RR}=$ 0.71 [95\% CI 0.50, 1.00]). Neither the Harbord test nor the funnel plot suggested publication bias (coefficient $=-0.733$, $\mathrm{SE}=0.828, p=0.410$; ESM Fig. 4a).

Among five placebo-controlled trials [24, 32, 36, 41, 42] ( $n=1,950$ vs 1,918$)$, the pooled RR was 0.45 (95\% CI 0.26 , $0.79)$ with moderate heterogeneity $\left(\chi^{2}=8.64, d f=4\right.$, $p=0.071, I^{2}=53.7 \%$; Fig. 2d). This suggested that ACEI/ ARB significantly reduced risk of macroalbuminuria by $55 \%$. The Harbord test suggested asymmetry of the funnel plot (coefficient $=-2.043, \mathrm{SE}=0.547, p=0.033$ ). The contourenhanced funnel plot showed that one-half of the studies included were in the high- and non-significance areas (ESM Fig. 4b), suggesting treatment-effect heterogeneity. Metaregression analysis fitting type of patient (microalbuminuria and mixed patients) reduced the degree of heterogeneity $\left(I^{2}\right)$ from $53.7 \%$ to $0 \%$. Subgroup analysis performed in patients with microalbuminuria and mixed micro- and macroalbuminuria yielded pooled RRs of 0.31 (95\% CI 0.18, 0.57) and 0.45 (95\% CI 0.26, 0.79), respectively.

Microalbuminuria Nine studies [19, 21, 22, 25, 26, 31, 35, $39,43]$ had microalbuminuria outcomes $(n=7,891)$ : six [19, $21,22,25,26,43]$ looking at ACEI vs other antihypertensive drugs, two looking at ACEI vs placebo [31, 43] and two looking at ARB vs placebo $[35,39]$ (ESM Table 5).
The pooled treatment effects between ACEI/ARB and other antihypertensive drugs were somewhat heterogeneous $\left(\chi^{2}=7.70, d f=5, p=0.174, I^{2}=35.1 \%\right.$ ) with a pooled RR of 0.84 (95\% CI $0.61,1.15)$ (Table 2 and Fig. 2e). The Harbord test suggested no asymmetry of the funnel plot (coefficient $=-0.281, \mathrm{SE}=1.367, p=0.847$; ESM Fig. 5a).

Compared with placebo in four studies [31, 35, 39, 43] ( $n=3,290$ vs 3,472), the pooled effect of ACEI/ARBs [24, 28, 32, 37] was 0.82 (95\% CI 0.64, 1.05; Fig. 2e) with moderate heterogeneity $\left(\chi^{2}=5.93, d f=3, p=0.115\right.$, $I^{2}=49.4 \%$ ). Meta-regression could not identify the source of the heterogeneity. The Harbord test suggested asymmetry of the funnel (coefficient $=-2.626, \mathrm{SE}=0.360, p=0.018$ ), and the contour-enhanced funnel plot suggested that this heterogeneity might be the cause of the asymmetry (ESM Fig. 5b).

Albuminuria regression Ten studies [18, 21, 25, 27-29, 36, $38,44,45]$ reported regression of albuminuria $(n=3,710$; ESM Table 6).

The treatment effect between ACEI/ARB and other antihypertensive drugs, based on nine studies [18, 21, 25, 27 , $28,36,38,44,45](n=646$ vs 640$)$, was homogeneous $\left(\chi^{2}=8.74, d f=8, p=0.365, I^{2}=8.4 \%\right)$ with a borderline non-significant effect of $16 \%$ (pooled $\mathrm{RR}=1.16$ [95\% CI 0.99, 1.39]; see Table 2 and Fig. 2f). Neither the Harbord test nor a funnel plot suggested publication bias (coefficient $=-0.086, \mathrm{SE}=0.718, p=0.907$; ESM Fig. 6). 
For the placebo-controlled trials ( $n=947$ vs 948$)$, the likelihood of albuminuria regression was $17 \%$ higher with $\mathrm{ACEI} / \mathrm{ARB}$, which was on the borderline of non-significance $(\mathrm{RR}=1.17$ [95\% CI 1.00, 1.37]; Table 2).

\section{Network meta-analysis}

ESRD A network meta-analysis was applied to assess all treatment comparisons, (ESM Fig. 7a and ESM Table 8). Arrows and tails refer to interventions and comparators, bold and dashed lines refer to direct and indirect comparisons, respectively. Numbers under the line refer to the number of studies for the direct comparator data, whereas numbers above the line indicate pooled RRs. The analysis suggested that the ACEI/ARB $(n=6,092)$ significantly decreased the risk of ESRD when compared with d-CCB $(n=644)$ and placebo $(n=5,569$,$) with pooled RRs of 0.25$ (95\% CI $0.07,0.96)$ and 0.77 (95\% CI $0.64,0.92)$, respectively. However, the risk of ESRD was $2.00(95 \%$ CI $0.35,11.55)$ times higher, but not significantly so, compared with the BB group $(n=411)$. Comparing ACEI/ARB with all antihypertensive drugs $(\mathrm{dCCB}+\mathrm{BB})$ resulted in $62 \%$ significantly lower risk of ESRD (pooled RR $=0.38$ [95\% CI 0.31, 0.47]).

Pooling mean BPs from four studies [21, 26, 30, 40] showed non-significant differences between groups, with mean differences of $1.27(95 \% \mathrm{CI}-1.42,3.95)$ for systolic BP (SBP) and $-0.71(95 \% \mathrm{CI}-3.88,2.46)$ for diastolic BP (DBP).

Doubling of serum creatinine ESM Fig. $7 \mathrm{~b}$ and ESM Table 8 show that ACEI/ARBs $(n=7,831)$ significantly reduced the risk of serum creatinine doubling by $77 \%$ (pooled $\mathrm{RR}=0.23$ [95\% CI 0.09, 0.57]), 72\% (pooled RR $=0.28$ [95\% CI 0.11 , $0.71]$ ), and $21 \%$ (pooled $\mathrm{RR}=0.79$ [95\% CI $0.75,0.84]$ ) compared with d-CCB $(n=567)$, nd-CCB/BB $(n=34)$, and placebo $(n=7,784)$, respectively. Combining all antihypertensive drugs $(n=601)$ and comparing with ACEI/ARB resulted in $77 \%$ significantly lower risk (pooled $R R=0.23$ [95\% CI 0.09, 0.58]).

Microvascular complications The number of participants in each treatment arm is described in ESM Table 3, with total number of patients in ACEI/ARB and placebo of 3,284 vs 2,847 , respectively. ACEI/ARBs significantly reduced the risk of microvascular complications by $19 \%$ (pooled $\mathrm{RR}=$ 0.81 [95\% CI 0.71, 0.92]) when compared with placebo, but increased the risk almost twofold when compared with BB (ESM Fig. 7c and ESM Table 8).

Macroalbuminuria Network meta-analysis was performed based on 12 studies $[19,21-26,32,36,38,41,42]$ $(n=5,151$; ESM Table 4). ACEI/ARBs $(n=2,580)$ significantly reduced macroalbuminuria by $29 \%$ (pooled $\mathrm{RR}=0.71$
[95\% CI $0.62,0.83])$ compared with BB/diuretic $(n=441)$, $55 \%$ (pooled $\mathrm{RR}=0.45$ [95\% CI $0.29,0.70]$ ) vs d-CCB $(n=212)$, and $33 \%$ (pooled RR $=0.67$ [95\% CI 0.49, 0.92]) vs placebo $(n=1,918$; ESM Fig. $7 \mathrm{~d}$ and ESM Table 8$)$. The ACEI/ARBs significantly reduced macroalbuminuria by $40 \%$ (pooled RR $=0.60$ [95\% CI $0.47,0.77]$ ) compared with all antihypertensive drugs $(n=653)$.

Pooling SBP and DBP in three studies [21, 38, 41], the mean SBP and DBP differences were 2.82 (95\% CI 0.32 , $5.31)$ and $0.33(95 \% \mathrm{CI}-0.93,1.60) \mathrm{mmHg}$, respectively.

Microalbuminuria Based on pooling of results from nine studies [19, 21, 22, 25, 26, 31, 35, 39, 43] $(n=7,891)$, ACEI/ ARB $(n=3,704)$ had significantly better benefit than d-CCB $(n=238)$ with a pooled RR of $0.50(95 \%$ CI $0.38,0.65)$, but significantly worse outcomes than BB (i.e. favouring BB ) with the pooled RR of 0.48 (95\% CI $0.35,0.66$; ESM Fig. 7e and ESM Table 8). When compared with nd-CCB $(n=303)$ and placebo $(n=3,472)$, ACEI/ARBs did not reduce microalbuminuria with pooled RRs of $0.93(95 \% \mathrm{CI}$ $0.71,1.22)$ and $1.01(95 \%$ CI $0.82,1.25)$, respectively. Compared with all antihypertensive drugs, the ACEI/ARB significantly reduced the risk by $39 \%$ (pooled $R R=0.61$ [95\% CI 0.45, 0.83]).

Regression of albuminuria Using data from ten studies $(n=3,710)[18,21,25,27-29,36,38,44,45]$, ACEI/ARB $(n=1,582)$ significantly promoted regression of albuminuria compared with placebo $(n=948)$, but not with diuretic $(n=283)$; RRs were $1.35(95 \%$ CI $1.07,1.70)$ in favour of ACEI/ARB and 1.28 (95\% CI 1.02, 1.60) in favour of diuretic, respectively (ESM Fig. 7f and ESM Table 8). The pooled RR was $1.16(95 \%$ CI $0.83,1.63)$ for $\mathrm{ACEI} / \mathrm{ARB}$ vs d-CCB $(n=357)$.

In six of ten studies where data were available [21, 27-29, 44, 45], the mean SBP was significantly higher for ACEI/ ARB (mean difference $=2.35[95 \%$ CI 1.07, 3.64]) than placebo but not so for DBP (mean difference $=0.54 \mathrm{mmHg}$ $[95 \% \mathrm{CI}-0.40,1.48 \mathrm{mmHg}])$.

\section{Discussion}

The direct and indirect comparisons in our study confirm the clear benefit of ACEI/ARB over placebo for all outcomes (ESRD, serum creatinine doubling, microvascular complications, macroalbuminuria and regression of albuminuria) except microalbuminuria. The lack of a statistically significant treatment effect for microalbuminuria outcome is likely to result partly from the heterogeneity of treatment effects across the studies.

Our study extends the results of Strippoli et al. [11], who showed borderline significant benefit for ACEI/ARB vs 
placebo for doubling of serum creatinine $(\mathrm{RR}=0.79[95 \%$ CI $0.75,0.84]$ vs 0.60 [95\% CI $0.34,1.05])$ and ESRD ( $\mathrm{RR}=0.77$ [95\% CI $0.64,0.92]$ vs 0.64 [95\% CI $0.4,1.03]$ ). The addition of new data and the use of network meta-analysis methods increase the power and allow us to demonstrate the reno-protective effects of RAS blockade more confidently.

The major contribution of our study is in teasing out the potential reno-protective effect of ACEI/ARBs over other antihypertensive drugs. Overall, the direct meta-analysis suggests a potential benefit of ACEI/ARB in reducing macroalbuminuria and doubling of serum creatinine, and in promoting regression of albuminuria. The results of network meta-analysis suggest additional benefits of the ACEI/ARB in reducing the risk of ESRD. These benefits contradict the previous meta-analysis [9], which found renoprotective effects of ACEI/ARB in patients without diabetes but not in patients who had already developed diabetic nephropathy. This discrepancy with previous work may be due partly to the addition of new data, thereby improving power, or may be driven partly by the fact that the major active comparators were CCBs; in some comparisons, BBs and diuretics are significantly better than ACEI/ARBs, though the numbers in these comparisons are small and driven largely by the UK Prospective Diabetes Study (UKPDS).

In the studies that reported BP change, there was no significant difference in BP decrease between ACEI/ARB and other antihypertensive drugs, suggesting that there is a specific reno-protective effect of ACEI/ARBs beyond their antihypertensive effect. This runs contrary to the conclusion of Casas et al. [9], who found the effect of RAS blockade to depend on its BP-lowering effect. In their study, Casas et al. showed that the benefit of RAS blockers compared with active control was associated with the extra degree of BP decrease. These results are hard to reconcile with summarylevel data. It is of course possible for both conclusions to be true, i.e. the effect of RAS blockers is linked to the level of BP control but that for two agents that bring about the same drop in BP, RAS blockers would have an additional benefit. This possibility would be better teased out with a metaanalysis of individual patient data or a direct test of this hypothesis in a large-scale RCT.

Our study has some strengths. We have applied a network meta-analysis to increase the power of the tests and reduce type I errors [15-17]. We applied a mixed model, which is thought to be the most appropriate method for this kind of pooling. The network method 'borrows' treatment information from other studies and increases the total sample size. As a result, treatment effects that could not be detected or treatment effects of borderline significance in direct metaanalysis could be identified. In addition, all possible treatment comparisons are mapped and displayed (see ESM Table 9). The weakness of our study is that despite the large overall numbers, some comparator groups still have small numbers, leading to poor precision of estimates. The lack of BP data for some studies also limits our ability to judge the specific reno-protective effects.

In conclusion, there appears to be a consistent benefit of ACEI/ARB on all outcomes in type 2 diabetes, including the 'hard clinical endpoints' of ESRD and doubling of serum creatinine, when compared with other antihypertensive drugs (mainly CCBs) and placebo, both in direct and indirect meta-analyses. This is seen in the context of no difference in BP decrease, suggesting a specific reno-protective effect. Other antihypertensives, particularly BBs and diuretics, may have even greater reno-protective effects but this needs to be investigated in further studies or individual patient meta-analysis.

Acknowledgements This study was funded by a grant from the Multi-Sectoral Network for Non-Communicable Disease Control (NCD network) of Thailand.

Contribution statement AT had full access to all of the data in the study and takes responsibility for the integrity of the data and the accuracy of the data analysis. PV, DL, AT and JA contributed to the study concept and design. AT and JA contributed to study supervision. PV, AT, DL and AI contributed to the acquisition of data. PV, AT, AI, $\mathrm{CN}$ and JA contributed to analysis and interpretation of data. PV, DL and AT contributed to the drafting of the manuscript. JA, AT, AI and $\mathrm{CN}$ contributed to the critical revision of the manuscript for important intellectual content. All authors gave final approval of the version to be published.

Duality of interest The authors declare that there is no duality of interest associated with this manuscript. The funding organisation had no role in the design or conduct of the study.

Open Access This article is distributed under the terms of the Creative Commons Attribution Noncommercial License which permits any noncommercial use, distribution, and reproduction in any medium, provided the original author(s) and source are credited.

\section{References}

1. Scheffel RS, Bortolanza D, Weber CS et al (2004) Prevalence of micro and macroangiopatic chronic complications and their risk factors in the care of outpatients with type 2 diabetes mellitus. Rev Assoc Med Bras 50:263-267

2. Ubink-Veltmaat LJ, Bilo HJ, Meyboom-de Jong B (2004) Microalbuminuria in patients with type 2 diabetes mellitus in general practice. Ned Tijdschr Geneeskd 148:2026-2030

3. Wu AY, Kong NC, de Leon FA et al (2005) An alarmingly high prevalence of diabetic nephropathy in Asian type 2 diabetic patients: the MicroAlbuminuria Prevalence (MAP) Study. Diabetologia 48:17-26

4. Gall MA (1997) Albuminuria in non-insulin-dependent diabetes mellitus. Prevalence, causes, and consequences. Dan Med Bull $44: 465-485$ 
5. Garg JP, Bakris GL (2002) Microalbuminuria: marker of vascular dysfunction, risk factor for cardiovascular disease. Vasc Med 7:3543

6. Klausen K, Borch-Johnsen K, Feldt-Rasmussen B et al (2004) Very low levels of microalbuminuria are associated with increased risk of coronary heart disease and death independently of renal function, hypertension, and diabetes. Circulation 110:32-35

7. Ravid M, Lang R, Rachmani R, Lishner M (1996) Long-term renoprotective effect of angiotensin-converting enzyme inhibition in non-insulin-dependent diabetes mellitus. A 7-year follow-up study. Arch Intern Med 156:286-289

8. Ruggenenti P, Perna A, Gherardi G, Benini R, Remuzzi G (2000) Chronic proteinuric nephropathies: outcomes and response to treatment in a prospective cohort of 352 patients with different patterns of renal injury. Am J Kidney Dis 35:1155-1165

9. Casas JP, Chua W, Loukogeorgakis S et al (2005) Effect of inhibitors of the renin-angiotensin system and other antihypertensive drugs on renal outcomes: systematic review and meta-analysis. Lancet 366:2026-2033

10. Kunz R, Friedrich C, Wolbers M, Mann JF (2008) Meta-analysis: effect of monotherapy and combination therapy with inhibitors of the renin angiotensin system on proteinuria in renal disease. Ann Intern Med 148:30-48

11. Strippoli GF, Craig M, Deeks JJ, Schena FP, Craig JC (2004) Effects of angiotensin converting enzyme inhibitors and angiotensin II receptor antagonists on mortality and renal outcomes in diabetic nephropathy: systematic review. BMJ 329:828

12. Sterne JA, Sutton AJ, Ioannidis JP et al (2011) Recommendations for examining and interpreting funnel plot asymmetry in metaanalyses of randomised controlled trials. BMJ 343:d4002

13. Peters JL, Sutton AJ, Jones DR, Abrams KR, Rushton L (2008) Contour-enhanced meta-analysis funnel plots help distinguish publication bias from other causes of asymmetry. J Clin Epidemiol 61:991-996

14. Palmer TM, Peter JL, Sutton AJ, Moreno SG (2008) Contourenhanced funnel plots in meta-analysis. Stata J 8:242-254

15. Lu G, Ades AE (2004) Combination of direct and indirect evidence in mixed treatment comparisons. Stat Med 23:3105-3124

16. Song F, Altman DG, Glenny AM, Deeks JJ (2003) Validity of indirect comparison for estimating efficacy of competing interventions: empirical evidence from published meta-analyses. BMJ 326:472

17. Song F, Harvey I, Lilford R (2008) Adjusted indirect comparison may be less biased than direct comparison for evaluating new pharmaceutical interventions. J Clin Epidemiol 61:455-463

18. Kashiwagi A (2007) Reduction of microalbuminuria in patients with type 2 diabetes. The Shiga Microalbuminuria Reduction Trial (SMART). Diabetes Care 30:1581-1583

19. Estacio RO, Jeffers BW, Gifford N, Schrier RW (2000) Effect of blood pressure control on diabetic microvascular complications in patients with hypertension and type 2 diabetes. Diabetes Care 23 (Suppl 2):B54-B64

20. Bakris GL, Copley JB, Vicknair N, Sadler R, Leurgans S (1996) Calcium channel blockers versus other antihypertensive therapies on progression of NIDDM associated nephropathy. Kidney Int 50:1641-1650

21. Chan JC, Ko GT, Leung DH et al (2000) Long-term effects of angiotensin-converting enzyme inhibition and metabolic control in hypertensive type 2 diabetic patients. Kidney Int 57:590-600

22. Lacourciere Y, Nadeau A, Poirier L, Tancrede G (1993) Captopril or conventional therapy in hypertensive type II diabetics. Threeyear analysis. Hypertension 21:786-794

23. Shiba T, Inoue M, Tada $H$ et al (2000) Delapril versus manidipine in hypertensive therapy to halt the type-2-diabetes-mellitus-associated nephropathy. Diabetes Res Clin Pract 47:97-104

24. Heart Outcomes Prevention Evaluation (HOPE) Study Investigators (2000) Effects of ramipril on cardiovascular and microvascular outcomes in people with diabetes mellitus: results of the HOPE study and MICRO-HOPE substudy. Lancet 355:253-259

25. Baba S (2001) Nifedipine and enalapril equally reduce the progression of nephropathy in hypertensive type 2 diabetics. Diabetes Res Clin Pract 54:191-201

26. UK Prospective Diabetes Study Group (1998) Efficacy of atenolol and captopril in reducing risk of macrovascular and microvascular complications in type 2 diabetes: UKPDS 39. BMJ 317:713-720

27. Fogari R, Mugellini A, Zoppi A et al (2005) Effect of successful hypertension control by manidipine or lisinopril on albuminuria and left ventricular mass in diabetic hypertensive patients with microalbuminuria. Eur J Clin Pharmacol 61:483-490

28. Fogari R, Preti P, Zoppi A et al (2002) Effects of amlodipine fosinopril combination on microalbuminuria in hypertensive type 2 diabetic patients. Am J Hypertens 15:1042-1049

29. Marre M, Lievre M, Chatellier G, Mann JF, Passa P, Menard J (2004) Effects of low dose ramipril on cardiovascular and renal outcomes in patients with type 2 diabetes and raised excretion of urinary albumin: randomised, double blind, placebo controlled trial (the DIABHYCAR study). BMJ 328:495

30. Nielsen FS, Rossing P, Gall MA, Skott P, Smidt UM, Parving HH (1997) Long-term effect of lisinopril and atenolol on kidney function in hypertensive NIDDM subjects with diabetic nephropathy. Diabetes 46:1182-1188

31. Ravid M, Brosh D, Levi Z, Bar-Dayan Y, Ravid D, Rachmani R (1998) Use of enalapril to attenuate decline in renal function in normotensive, normoalbuminuric patients with type 2 diabetes mellitus. A randomized, controlled trial. Ann Intern Med 128:982-988

32. Ravid M, Savin H, Jutrin I, Bental T, Katz B, Lishner M (1993) Long-term stabilizing effect of angiotensin-converting enzyme inhibition on plasma creatinine and on proteinuria in normotensive type II diabetic patients. Ann Intern Med 118:577-581

33. Lewis EJ, Hunsicker LG, Clarke WR et al (2001) Renoprotective effect of the angiotensin-receptor antagonist irbesartan in patients with nephropathy due to type 2 diabetes. N Engl J Med 345:851860

34. Brenner BM, Cooper ME, de Zeeuw D et al (2001) Effects of losartan on renal and cardiovascular outcomes in patients with type 2 diabetes and nephropathy. N Engl J Med 345:861-869

35. Bilous R, Chaturvedi N, Sjolie AK et al (2009) Effect of candesartan on microalbuminuria and albumin excretion rate in diabetes: three randomized trials. Ann Intern Med 151(11-20):W13-W14

36. Jerums G, Allen TJ, Campbell DJ et al (2004) Long-term renoprotection by perindopril or nifedipine in non-hypertensive patients with type 2 diabetes and microalbuminuria. Diabet Med 21:11921199

37. Sjolie AK, Klein R, Porta M et al (2008) Effect of candesartan on progression and regression of retinopathy in type 2 diabetes (DIRECT-Protect 2): a randomised placebo-controlled trial. Lancet 372:1385-1393

38. Marre M, Puig JG, Kokot F et al (2004) Equivalence of indapamide SR and enalapril on microalbuminuria reduction in hypertensive patients with type 2 diabetes: the NESTOR Study. J Hypertens 22:1613-1622

39. Haller H, Ito S, Izzo JL Jr et al (2011) Olmesartan for the delay or prevention of microalbuminuria in type 2 diabetes. N Engl J Med 364:907-917

40. Fogari R, Zoppi A, Corradi L et al (1999) Long-term effects of ramipril and nitrendipine on albuminuria in hypertensive patients with type II diabetes and impaired renal function. J Hum Hypertens 13:47-53

41. Ahmad J, Siddiqui MA, Ahmad H (1997) Effective postponement of diabetic nephropathy with enalapril in normotensive type 2 diabetic patients with microalbuminuria. Diabetes Care 20:15761581 
42. Lebovitz HE, Wiegmann TB, Cnaan A, et al. (1994) Renal protective effects of enalapril in hypertensive NIDDM: role of baseline albuminuria. Kidney Int (Suppl 45):S150-S155

43. Ruggenenti P, Fassi A, Ilieva AP et al (2004) Preventing microalbuminuria in type 2 diabetes. N Engl J Med 351:1941-1951

44. Mosconi L, Ruggenenti P, Perna A, Mecca G, Remuzzi G (1996) Nitrendipine and enalapril improve albuminuria and glomerular filtration rate in non-insulin dependent diabetes. Kidney Int Suppl 55:S91-S93

45. Ogawa S, Takeuchi K, Mori T, Nako K, Tsubono Y, Ito S (2007) Effects of monotherapy of temocapril or candesartan with dose increments or combination therapy with both drugs on the suppression of diabetic nephropathy. Hypertens Res $30: 325-334$ 